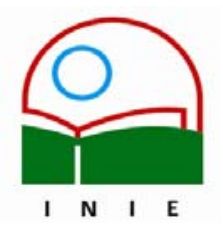

Universidad de Costa Rica

Facultad de Educación

Instituto de Investigación en Educación

ACTUALDADES INVESTIGATIVASEN EDUCACION

\title{
VIVENCIAS DE LA EXPRESIÓN MUSICAL: ÁREAS Y ESTRATEGIAS METODOLÓGICAS
}

\author{
EXPERIENCES OF MUSICAL EXPRESSION: METHODOLOGICAL AREAS \\ AND STRATEGIES
}

\begin{abstract}
Consuelo Arguedas Quesada ${ }^{1}$
"Hacer tu propia música es claramente relativo a la cultura musical a la que se ha estado expuesto: étnica, religiosa, folklórica, popular y todas las subculturas intermedias" (Glover, 2004, p. 42)
\end{abstract}

Resumen: En el siguiente artículo se analizan las áreas de la educación musical: audición, rítmica, canto, lectoescritura, ejecución instrumental y apreciación musical.

Se plantea la importancia de estimular la audición mediante ejercicios relacionados con sonidos agudos, graves y centrales, giros melódicos, sonidos "sol, mi, do" y timbres sonoros; en el desarrollo rítmico se enfatiza en el pulso, el acento , el ritmo y el ostinato; en el canto se consideran las opciones de trabajarlo mediante cambios de velocidad, intensidad, con preguntas y respuestas, utilizando el canto en cadena, los juegos con nombres, los saludos cantados, la audición interior, los cantos para estimular la creación, los juegos para fortalecer el diafragma, la respiración y la articulación, así como también el canon; en la lecto-escritura y ejecución musical se sugiere la posibilidad de basarse en métodos específicos ya establecidos, o poner en práctica partituras no convencionales que se basan en dibujos, pinturas o diversos gráficos; en la apreciación musical se enfatiza en la necesidad de recurrir a obras de distintos autores, estilos y periodos para ofrecer una gama amplia en el repertorio que se utilice.

Además de las variadas estrategias metodológicas para el trabajo de cada una de las áreas mencionadas, se proporcionan ideas para la confección de instrumentos musicales y materiales auxiliares con objetos de desecho.

Palabras clave: ÁREAS DE LA EDUCAICÓN MUSICAL, ESTRATEGIAS METODOLÓGICAS, CREACIÓN DE MATERIALES E INSTRUMENTOS

\begin{abstract}
In the following article the following areas of musical education are analyzed: hearing, rhythm, song, reading \& writing, performing with an instrument and musical appraisal. The author stresses the importance of stimulating hearing through exercises related to high-pitched, low-pitched and central-pitched sounds, melodic twists, "sol-mi-do" sounds and timbres. When developing rhythm; pulse, accent, rhythm and ostinato are emphasized. In song, several possibilities for its exercise are considered, such as changes in speed, intensity, questions and answers, chained songs, wordplay with names, sung greetings, interior hearing, singing to stimulate creation, games to strengthen the diaphragm, respiration and articulation, as well as the canon. In reading \& writing and in musical performance the possibility of using well-stablished principles is suggested, or using non-conventional musical notation such as music represented by drawings, paintings or a diversity of graphics. When teaching to appraise music, the necessity for considering works from many different authors, styles and periods in order to offer a wide gamut in the repertoire to be studied.

In addition to the varied methodological strategies given for the labor in each of the mentioned areas, some ideas for the construction of musical instruments and other auxiliary materials with discarded objects are also included.
\end{abstract}

Keywords: AREAS IN MUSICAL EDUCATION, METHODOLOGICAL STRATEGIES, CREATION OF MATERIALS AND INSTRUMENTS

\footnotetext{
${ }^{1}$ Licenciada en Música con énfasis en Educación Musical de la Universidad Nacional, Costa Rica. Profesora Especializada del Programa Superior de Perfeccionamiento en Educación Musical del Instituto Interamericano de Educación Musical, Universidad de Chile. Profesora de la Escuela de Formación Docente, Depto. de Primaria y Preescolar, y Depto. de Educación Secundaria; Investigadora del Instituto de Investigación en Educación (INIE) de la Universidad de Costa Rica. Correo electrónico: consueloa@racsa.co.cr
}

Artículo recibido: 21 de junio, 2006

Aprobado: 4 de diciembre, 2006 


\section{Introducción}

El siguiente artículo surge a raíz de las consultas bibliográficas y de mi experiencia como docente de educación musical obtenida en las aulas, en los talleres realizados en diferentes contextos educativos, durante los proyectos de Acción Social y de Investigación, así como también como complemento del Proyecto UNESCO "La expresión artística. Un medio para construir procesos pedagógicos en el aula".

Para iniciar mi deliberación sobre el tema escogido, creo firmemente en que la educación musical ofrece múltiples posibilidades para "hacer música", vivenciarla e interiorizarla. Su enseñanza y aprendizaje no se basan tan sólo en aspectos teóricos, memorísticos o repetitivos, por el contrario, las diversas áreas permiten incursionar de manera activa, según la experiencia de cada persona, estimulando la libre expresión y las posibilidades sonoras del cuerpo. Es decir, "saber música", engloba un enfoque amplio, donde la población estudiantil tenga acceso a todo tipo de música, experimente con los sonidos y los ruidos y por ende, con el empleo de grafías no convencionales, así como también con la expresión rítmica, melódica, armónica, instrumental, vocal y corporal.

Desde la música, el niño y la niña perciben el entorno sonoro como parte de su medio, son auditores e intérpretes y se convierten en realizadores expresivos y creativos que se acercan a la vida musical experimentándola de forma activa, mediante el uso de la voz, el oído musical, el movimiento asociado a la música y las habilidades instrumentales. (Pascual, 2002, p. 52)

La relación que se establece en la niñez entre los objetos y el juego, ya sea individualmente o con otros niños y niñas, convierte al sonido en un puente entre la fantasía y la realidad. El tocar los juguetes o distintos materiales, golpearlos y experimentar sonidos diversos, permite que éste sea de gran importancia en sus actividades diarias al convertir las imágenes en realidad. "La cualidad organizativa de la audición le permite distinguir, reconocer y ordenar su percepción del entorno" (Alsina, 1999, p. 7).

Es por esta razón, que para vivenciar la expresión musical, se estimulan las áreas de la audición, la rítmica, el canto, la lecto-escritura, la ejecución instrumental y la apreciación musical, mediante estrategias interesantes de manera lúdica. 


\section{1. Áreas de la expresión musical y estrategias metodológicas}

\subsection{Audición}

La audición se desarrolla al poner en práctica ejercicios para discriminar la altura del sonido y el timbre sonoro. Otros ejercicios consisten en ubicar el sonido, ya sea dentro, fuera, lejos, cerca, los que son agradables, los desagradables o los estacionarios (se escuchan en un mismo lugar por un tiempo determinado).

\subsubsection{Altura del sonido}

Según Pascual (2002, p. 294)," la altura es la frecuencia o número de vibraciones por segundo, que musicalmente da lugar a los intervalos, los tonos, los semitonos, la aparición de la melodía y la armonía".

Para discriminar la altura del sonido, se realizan prácticas para identificar los sonidos que son agudos, graves y centrales o intermedios, así como también giros melódicos y series con los sonidos "sol y mi", "sol, mi y do".

a) Los sonidos agudos graves y centrales se pueden discriminar mediante instrumentos melódicos, es decir, aquellos que permiten interpretar melodías. Como por ejemplo la flauta, la guitarra, el acordeón, la organeta o el piano

- Una actividad puede ser que los estudiantes al escuchar los agudos den palmadas y con los graves percutan con los pies.

b) Posteriormente, se introduce un tercer elemento que es el sonido central, es decir, es intermedio entre el agudo y el grave; en este caso se reacciona con una percusión corporal central; puede ser palmadas sobre los muslos.

Los planos corporales que se utilicen pueden modificarse, siempre y cuando se respete la relación arriba - sonido agudo, intermedio - sonido central y abajo - sonido grave.

- Una vez interiorizados estos conceptos mediante la vivencia corporal, se procede a identificar los sonidos con símbolos. Puede ser con figuras realizadas con fieltro que se colocan en franelógrafos; también con cartulina o con foam para trabajar sobre el pupitre u otra superficie. 
Los símbolos para identificarlos son:

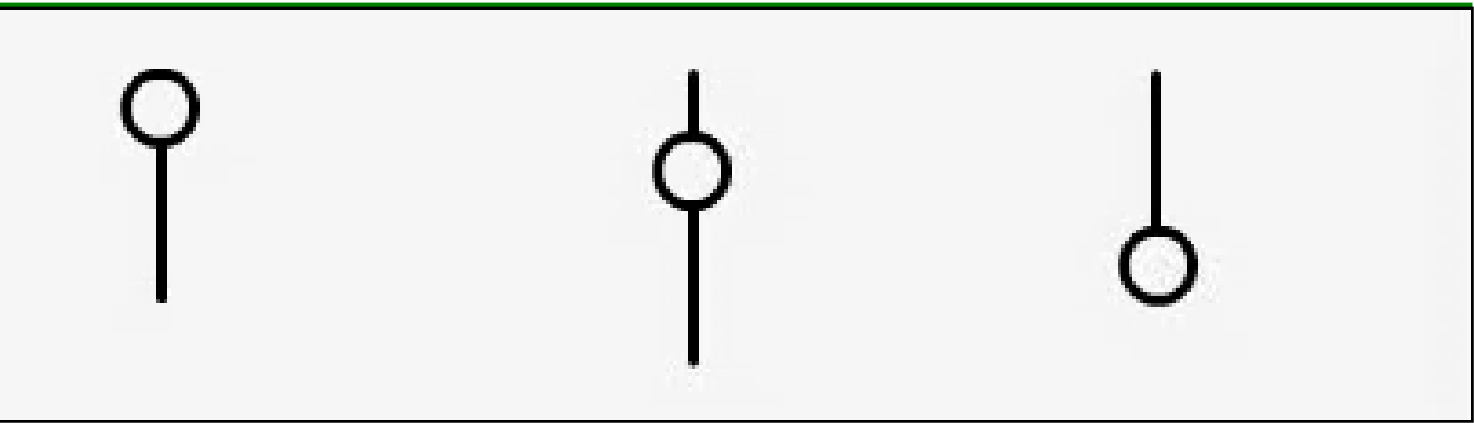

AGUDO

CENTRAL

GRAVE

Mediante la ejecución de dictados de series de sonidos donde se mezclen estas posibilidades, los estudiantes las reproducen, haciendo uso de las figuras previamente entregadas.

c) Los giros melódicos son series de cinco sonidos (do, re, mi, fa, sol) u ocho (do, re,mi, fa, sol, la, si, do agudo), que se interpretan de manera ascendente, descendente o repitiendo algunos de ellos. Para iniciar esta práctica, conviene hacerlos sólo con cinco sonidos, teniendo en cuenta que los que se repiten son el "do", el "mi" y el "sol" (se ejecutan tres veces el mismo sonido)

- Para interiorizar este contenido, se representa corporalmente si los sonidos suben, bajan o se mantienen; esto es poniéndose de pie o de puntillas si son agudos, agachándose hasta estar en contacto con el suelo cuando se trata de sonidos graves, o colocándose de cuclillas al escuchar los intermedios. También se puede indicar la dirección de los sonidos, al subir, bajar o mantener el brazo en determinada altura.

- Otra opción para reconocer los giros melódicos consiste en hacer uso de un elástico cerrado que se prensa con el pie sobre el piso. Éste se estira, se encoge o se mantiene en un misma posición según sea el estímulo sonoro. Se debe tener mucho cuidado en que esté bien sujetado, para no producir un golpe a la persona que lo manipula.

- Los y las estudiantes pueden reconocer también el giro melódico que se escucha, haciendo uso de tarjetas, juegos de bingo, o dibujos de trazos que indican la dirección del sonido.

- Una vez escuchada la serie de sonidos, se procede a dibujar el trazo respectivo. 
Algunos ejemplos de giros melódicos son los siguientes:
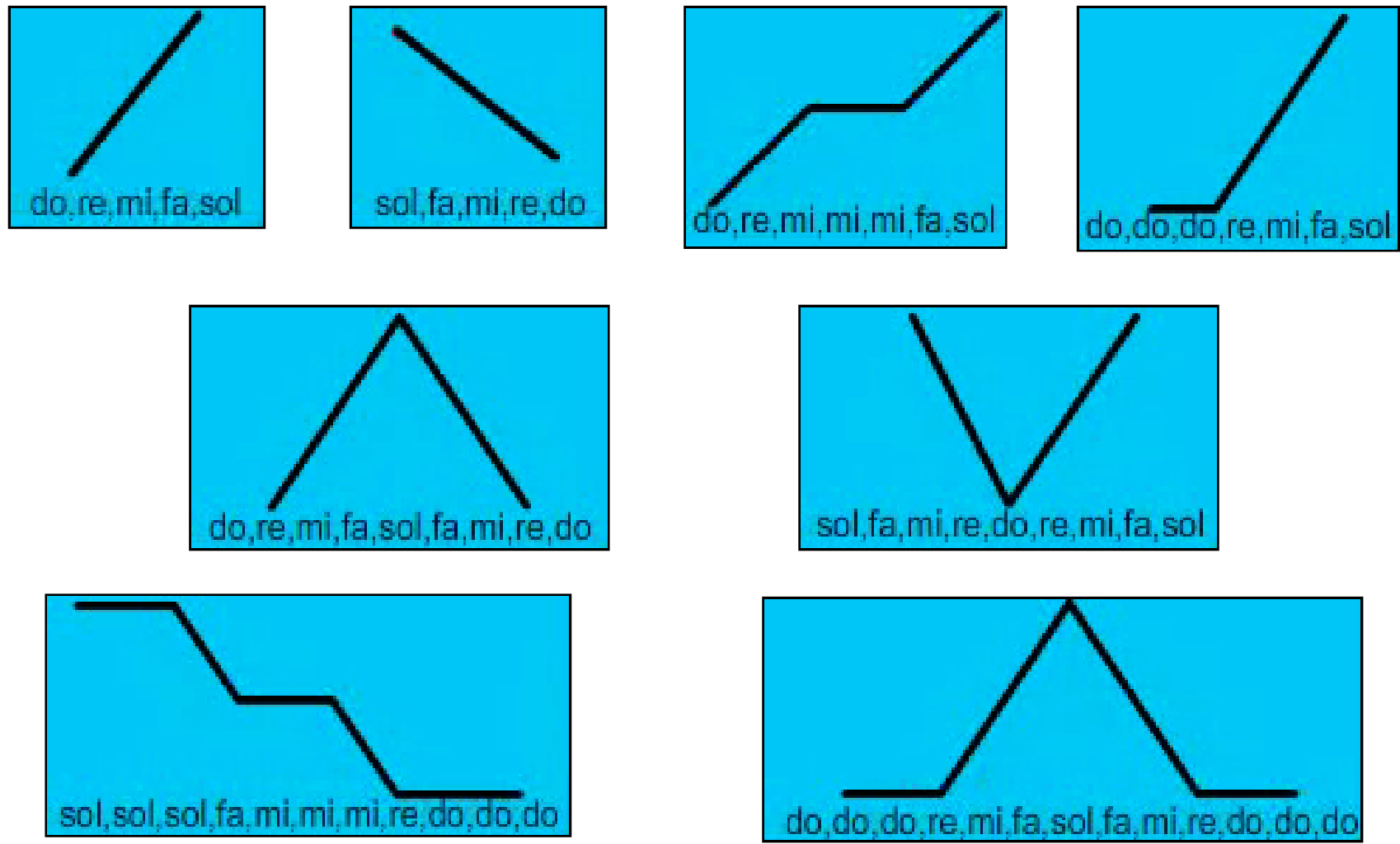

d) Dictados con "sol y mi", "sol, mi y do"

En este caso los ejercicios se aplican a personas que anteriormente han tenido un aprestamiento melódico mayor. Consiste en discriminar estos sonidos para ubicarlos en un bigrama (las dos líneas inferiores de un pentagrama), o directamente en un pentagrama (cinco líneas). Para iniciar este proceso se recomienda la primera opción, ya que la cuarta y la quinta línea del pentagrama con sus respectivos espacios aún no se utilizan.

\subsubsection{Timbre sonoro}

Según el Diccionario de la Real Academia (1964, p.1309), el término timbre corresponde al "modo propio y característico de sonar un instrumento musical o la voz de una persona"; de igual manera, este concepto se aplica al sonido que producen los objetos al moverse, sacudirse, frotarse, o al ser golpeados; otra posibilidad está en los sonidos específicos de algunas actividades, así como el ambiente sonoro que nos rodea. 
El compositor contemporáneo Murray Schafer, es un estudioso en este campo y se considera un ecologista que aprecia la vida y por lo tanto la ecología acústica; su preocupación es la contaminación sonora, lo que él denomina "el paisaje sonoro", en el que intervienen sonidos humanos, tecnológicos y de la naturaleza.

-En el trabajo de aula este tema se puede desarrollar, dentro de los contenidos del área de Ciencias como parte de la contaminación ambiental, mediante diversas actividades que permitan concientizar a los estudiantes, de que ésta representa una amenaza para los habitantes de nuestro planeta.

-Otros aspectos importantes relacionados con el timbre sonoro para trabajar con los y las estudiantes, son aquellos que facilitan su estimulación auditiva. En este caso, los sonidos producidos por instrumentos musicales, los sonidos procedentes de objetos, las voces de los compañeros y compañeras, sonidos de la comunidad, sonidos del entorno educativo, entre otros.

-Si se pone en práctica el uso de instrumentos musicales, se pueden ejecutar algunos conocidos por ellos para que los identifiquen. En este caso, es útil integrar tarjetas con los dibujos de los mismos, así como también figuras elaboradas con "foam" o fieltro, o trabajar con juegos de bingo, para que la población estudiantil los clasifique, los ordene o muestre, según el orden en que los escucha. Conviene escuchar los timbres de los instrumentos antes de realizar los dictados.

Por ejemplo: para el sonido del triángulo se puede realizar la siguiente figura para el de la flauta I, para el tambor o el pandero $\bigcirc$, y para la maraca

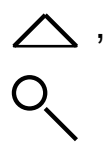

-También puede ser que se coloque a los estudiantes en tres círculos. Los integrantes de cada uno de ellos deben reaccionar ante el sonido de un instrumento específico que escuchen; ya sea dando palmadas, girar, saltar u otra actividad previamente indicada.

\subsection{Educación rítmica}

En esta área se involucran el ritmo y el movimiento, mediante la ejecución de instrumentos o el trabajo corporal. Comprende el pulso, el acento, el ritmo y el ostinato. 
El pulso de la música es la constante, el siempre igual; puede variar la velocidad al ejecutarlo pero no la duración, ya que no presenta diferencias entre sonidos cortos o largos, pues todos son iguales. Una manera de ejemplificarlo a los y las estudiantes es mediante el latido del corazón o el tic tac del reloj. Se aconseja iniciar su aprendizaje caminando o palmeándolo a canciones o melodías que se escuchan.

El acento corresponde a los pulsos fuertes de la música, que transcurren cada dos, tres, cuatro, o seis tiempos. Es aconsejable iniciar su aprendizaje, una vez que la vivencia del pulso esté interiorizada por los y las estudiantes.

El ritmo es equivalente a los sonidos largos, cortos o ausencia de ellos, es decir, concierne a las subdivisiones de los tiempos de la música. Si se entona una canción, su ritmo coincide con cada sílaba del texto. Por esta razón, las poesías, las rimas, las adivinanzas y los trabalenguas son muy útiles para percutir el ritmo.

El ostinato corresponde a un esquema rítmico o melódico que se repite de manera constante, sin modificarse y sirve como acompañamiento.

El pulso, el acento, el ritmo y el ostinato se pueden vivenciar con diversas posibilidades de movimientos corporales, así como también mediante timbres sonoros producidos por el cuerpo; también se realiza con instrumentos musicales. Algunos ejemplos pueden ser:

- Dar chasquidos indicando los acentos de una melodía que se escucha.

- Marcar el ritmo de una canción al percutir con dos dedos sobre la palma de la otra mano, los muslos, la frente o cualquier otra parte del cuerpo.

- Dar palmadas de manera alternada con otro compañero o compañera para vivenciar el pulso.

- Decir una frase rítmicamente, mientras el grupo entona una canción.

\subsubsection{Materiales auxiliares para trabajar el movimiento corporal}

Los materiales auxiliares que a continuación se mencionan tienen cada uno de ellos características especiales que permiten interiorizar diversos elementos de la expresión corporal y musical. No obstante, todos ofrecen múltiples opciones para ser utilizados como parte de una escenografía, ya sea para reconstruir un ambiente acorde con la actividad que se va a representar, o como elementos del vestuario de los participantes. 
El pulso, el acento, el ritmo, el ostinato, la velocidad, la intensidad, la forma y otras características de la música se vivencian de manera corporal para interiorizar estos conceptos, y al mismo tiempo se ponen en práctica elementos de la expresión corporal, como son los movimientos naturales (no ofrecen mayor complicación y la persona sana los realiza sin complicación alguna, por ejemplo caminar, correr, saltar); los movimientos analíticos o técnicos (se relacionan con alguna parte específica del cuerpo, por ejemplo las rotaciones, los estiramientos, las flexiones, las ondulaciones, las contracciones); y los movimientos generadores (indican la calidad de los movimientos, es decir pueden ser fuertes, suaves, rápidos, lentos y al mismo tiempo incluyen los naturales y los analíticos).

Resulta de gran utilidad integrar materiales auxiliares al trabajo corporal, ya que permiten reforzar el contenido musical en estudio. Algunos de ellos se mencionan a continuación:

\section{- Bolsito}

Se confecciona con dos trozos de tela cosidos de $13 \mathrm{~cm}$ de lado. Este bolsito se rellena aproximadamente hasta la mitad con alguna clase de granos (maíz, frijoles duros, maíz de palomitas, lágrimas de San Pedro, u otros que al percutirlos produzcan un sonido fuerte). Es importante que no esté pesado ni demasiado lleno porque su contenido no produce el sonido adecuado. La tela no puede ser gruesa porque no permite que se expanda su sonoridad, y se recomienda que sea un estampado o de un solo tono, pero no muy claro ni demasiado oscuro; es decir que sea agradable y que invite a manipularlo.

Son útiles para vivenciar el pulso y el acento musical

- Pañuelos

El color y la tela de los mismos queda a elección del estudiante; lo importante es que su tamaño no sea pequeño y que permita el movimiento con libertad. Este material es apropiado para trabajar sensibilización, niveles alto, medio y bajo de la expresión corporal, pulso, acento, ostinato, intensidad, velocidad.

- Cintas de tela

Dos cintas del color y el ancho que deseé el estudiante, con una longitud equivalente a la distancia existente entre el suelo y la cintura de la persona que las utilizará. Al igual que los pañuelos, éstas son aptas para ejercicios de sensibilización, clases de movimiento, niveles, pulso y acento. 
- Bolsas plásticas de basura

Se utilizan enteras o recortadas en tiras sin que se despeguen completamente (mantener completo el fondo de la bolsa o parte pegada).

Representan un material apropiado para obtener diferentes timbres sonoros, dependiendo de la manera de sacudirlas, "pellizcarlas", frotarlas o golpearlas, ya sean infladas o desinfladas. Además permiten ejecutar el pulso, el acento, el ritmo y el ostinato, clases de movimiento, niveles, espacios, velocidades, intensidades.

- Bastón

Confeccionados con palos de escoba del tamaño de la distancia comprendida entre la cintura de la persona que lo va a utilizar y el piso.

Es apropiado para la vivencia de los niveles de la expresión corporal, el espacio parcial, el pulso, el acento, el ritmo y el ostinato

- Aro

Elaborado con un pedazo de manguera de aproximadamente 70 centímetros de diámetro. Se introduce un trozo de madera en uno de los extremos, y posteriormente en el otro; de esta manera quedan ambos unidos. Para dar más resistencia se les sujeta con cinta adhesiva resistente. Otra opción de este material es el "hula hula" que se obtiene en el mercado.

El aro es muy útil para actividades relacionadas con espacio parcial y total, niveles pulso, acento, ostinato.

- Tela grande

Puede utilizarse un mantel o una sábana. Este material es conveniente para vivenciar el espacio total, parcial, nociones espaciales (dentro, fuera, sobre, debajo, lejos, cerca), pulso, acento.

\subsection{Canto}

La canción es un elemento importante en la experiencia musical, ya que en ella se reúnen la melodía, el ritmo y la armonía. Para Hemsy de Gainza citado por Muñoz (2002, p. 47), “la canción infantil es el alimento musical más importante que recibe el niño y la niña; al igual 
que para Willems los cantos constituyen una actividad sintética que engloban la sensibilidad, el ritmo, la melodía y la armonía"

En la canción está presente la audición y la posibilidad de ser interpretada por instrumentos o acompañada con movimientos corporales y gestos. Además mediante el canto, adquiere gran importancia la dimensión social, afectiva y comunicativa de los estudiantes, ya que permite exteriorizar sentimientos, estados de ánimo, se favorece la desinhibición, la integración y el deseo de compartir.

Algunas personas sufren de ciertas perturbaciones vocales al hablar y al cantar, ocasionándoles falta de sonoridad o un registro inadecuado (extensión vocal de sonidos agudos y graves). En este caso, se puede recurrir a ejercicios que corrigen estos problemas, aunque también son estrategias metodológicas útiles para la enseñanza del canto; éstas son: prácticas vocales con cambios de velocidad e intensidad, preguntas y respuestas, canto en cadena, eco melódico, juegos con nombres, saludos cantados, audición interior, canciones para estimular la creación, canon, juegos para fortalecer el diafragma, la respiración y la articulación.

\subsection{Canto con cambios de velocidad}

Conviene partir del canto a velocidad normal, como se realiza siempre; luego la misma canción se entona de forma lenta y posteriormente se hace rápido. Al iniciar esta estrategia, es importante que se interprete completa la canción, sin cambios de velocidad durante el transcurso de la misma. Una vez que se ha vivenciado la experiencia, pueden hacerse ciertas variantes siguiendo una indicación del docente, que previamente se ha explicado; puede ser por ejemplo, el movimiento rápido o lento de la mano de la persona que guía la actividad.

\subsubsection{Canto con cambios de intensidad}

En este caso la estrategia es similar a la anterior, teniendo en cuenta que lo que se modifica es cantar fuerte o suave y la indicación del docente puede ser cerrando la mano y abriéndola. 


\subsubsection{Preguntas y respuestas}

Esta actividad se lleva a cabo con canciones, pues se trabaja a partir de las frases del texto y por ende las frases musicales. Consiste en que el o la docente canta una frase y la población infantil entona la siguiente; así hasta finalizar. El procedimiento posterior es a la inversa, inician la canción los estudiantes y el docente canta la siguiente frase. Dicha estrategia permite verificar el grado de aprendizaje de las canciones.

Una variante puede ser al incluir cambios en la intensidad o la velocidad, es decir, al cantar una frase suave y la siguiente fuerte; o una frase rápida y la otra lenta.

\subsubsection{Canto en cadena}

En este caso, cada grupo o cada niño o niña cantan una frase de una canción, hasta concluirla. Por ejemplo: se divide a los estudiantes en cuatro filas o rondas y cada una de ellas entona una frase del canto. Es importante la concentración y la vivencia anterior de preguntas y respuestas.

\subsubsection{Eco melódico}

Consiste en repetir esquemas melódicos sencillos mediante el canto; esta estrategia permite desarrollar el oído musical y el registro vocal de los y las estudiantes.

\subsubsection{Juegos con nombres}

Esta actividad se puede realizar al pasar la lista de asistencia, lo que varía es que se dicen los nombres de los estudiantes de manera cantada. Esto es, el o la docente improvisa pequeñas melodías y la población estudiantil le responde cantando si la persona que ha sido llamada se encuentra presente $o$ ausente.

\subsubsection{Saludos cantados}

Como el término lo indica, los saludos de buenos días, buenas tardes, de despedida o bienvenida se realizan improvisando melodías.

\subsubsection{Audición interior}

Se le puede llamar "canto hacia adentro" o "guardar la canción", y consiste en cantar una parte y otro segmento tan sólo se piensa; por ejemplo, el docente indica con la mano abierta el momento en que se debe cantar y cuando la cierra, los estudiantes la siguen con el 
pensamiento. Continúan cantando de nuevo cuando se abra la mano del profesor o profesora.

Este ejercicio conviene llevarlo a la práctica después del juego de intensidad.

\subsubsection{Canciones para estimular la creación}

Algunos cantos ofrecen la posibilidad de modificar una palabra por otra o variar la idea que se desea expresar en el texto. En este caso, las canciones promueven la creación entre los y las estudiantes al ampliar el contenido de las mismas o sugerir otras propuestas interesantes. Por ejemplo, a partir del canto "Tengo un zapatito":

Tengo un zapatito lerila

tengo un zapatito leré

mediecitas de seda lerila

y una flor en el pié leré

Con mi zapatito lerila

con mi zapatito leré

bailaré una danza lerila

al estilo escocés leré

\section{Reproducir canción}

Se puede generar otro texto:

Tengo una granja lerila

tengo una granja leré

muchos animalitos lerila

y un granero también leré

En mi granja hay vacas lerila

en mi granja hay cerdos leré

hay también gallinitas lerila

que me dan de comer leré

\section{Reproducir canción}

\subsubsection{Juegos para fortalecer el diafragma}

- Algunos de ellos pueden ser el imitar sonidos de un tren en movimiento articulando el sonido ch-ch ch, de manera enérgica mientras se expulsa aire. 
- Reproducir el sonido de una máquina encendida con el sonido "p, p, p"; puede realizarse con vibración de los labios o sin ella.

- Imitar el sonido que se produce cuando se expulsa vapor, mediante el sonido "s" o "f".

\subsubsection{Canon}

Según el Diccionario de la Real Academia Española (1984, p. 258), este término corresponde a "una composición de contrapunto en que sucesivamente van entrando las voces, repitiendo o imitando cada una el canto de la que antecede, donde las diversas voces repiten sucesivamente lo que cantaron las anteriores". Un ejemplo de canon corresponde a "El cucú", cuyo texto dice así:

(1) En el lejano bosque

ya canta el cucú,

(2) oculto en el follaje

al búho contestó

Cucú lo llamó, cucú lo llamó,

cucú, cucú, cucú.

Cucú lo llamó, cucú lo llamó,

cucú, cucú, cucú.

El procedimiento para cantarlo es el siguiente:

- Se divide el grupo en dos subgrupos

- Se canta completa la canción

- Siguiendo la indicación del docente, entona la canción sólo el subgrupo que se le indica (en este caso empieza a cantarse donde aparece el número (1)

- Cuando el subgrupo que inició el canto va a pronunciar la palabra "oculto" (2), comienza a cantar un segundo grupo desde el principio, y el primero continúa con lo que le corresponde del texto hasta terminar, es decir, no se detiene ni entona lo del segundo.

- El número de veces que cada subgrupo empiece la canción, dependerá de la indicación que les dé el docente, así como también el momento en que deben detenerse.

- Por lógica, el subgrupo de estudiantes que empieza el canon, será el que termine de primero y el que inicia posterior será el último. 
- Cuando se vivencie el canon a dos voces puede realizarse a tres o cuatro, siguiendo las indicaciones anteriores.

\subsubsection{Juegos para fortalecer la respiración}

- Soplar para apagar una candela inexistente

- Inspirar un perfume agradable imaginario

- Inflar un globo irreal poco a poco, hasta convertirse en gigantesco

- Desinflar el globo inmenso, produciendo el sonido s-s-s. Al principio se sugiere expulsar el aire fuerte y luego se va disminuyendo la intensidad hasta quedar sin aire.

\subsubsection{Juegos para fortalecer la articulación}

Para que los y las estudiantes corrijan su articulación al cantar y al hablar, conviene que el docente dé el ejemplo. Es importante no hacerlo fuerte, ni demasiado rápido, pronunciar las palabras con nitidez y gesticulando de manera correcta.

Resultan oportunos los trabalenguas (juego de palabras que expresan algún mensaje) y las jitenjáforas (juego de palabras sin un sentido definido) En ambos casos se sugiere decirlas, pronunciando de manera correcta cada una de las palabras, esto es, abriendo la boca para que salgan los sonidos y gesticulando lo necesario. Ejemplos de trabalenguas pueden ser los siguientes:

- Compadre, cómpreme coco.

-Compadre, coco no compro

porque como poco coco como,

poco coco compro.

- La cara señuda y rara del sastre

que era un desastre,

no era una cara de sastre

sino un desastre de cara.

- Tucu tucu tucu turo

pesa adrede un palo duro

duro palo, palo duro

palo duro, duro palo

pesa tucu tucu turu. 
- $\quad$ Podador que mis parras podas, podas mis parras o tus parras podas?

-Ni podo tus parras ni mis parras podo, que podo las parras del tío Gasporro.

Ejemplos de jitenjáforas son:

- Chinela ela de la chinchinela

ela chin, ela chan,

ela chin, chen, chan

- Ententina sóbalo cabina

sóbalo catí catoca

eli beli bon ${ }^{2}$

Sugerencias para la enseñanza de una canción

Al seleccionar el repertorio vocal de la población estudiantil, conviene que la melodía no ofrezca grandes saltos y que se localice dentro de su tesitura; el texto debe coincidir con los intereses del grupo, según el nivel en que se encuentre; es importante que el ritmo ofrezca combinaciones de valores interesantes pero que no resulten complicadas.

Otros aspectos musicales que se deben considerar son la métrica $(2 / 4,3 / 4,4 / 4,6 / 8)$ y la tonalidad (se sugieren Do, Sol, Re y Fa mayor, La, Re menor, entre otras).

Es importante comenzar con cantos de melodías y ritmos sencillos, incorporando canciones folklóricas, rondas y juegos tradicionales.

Existen variadas maneras para enseñar una canción; una de ellas es la siguiente:

- La o el docente entona completa la canción.

- Se puede ilustrar el contenido de la misma mediante alguna lámina, fotografía, dibujo u otros.

- Si se conoce, se menciona el autor de la misma, brindando alguna información adicional de interés.

- Se comenta el contenido del texto.

${ }^{2}$ Los trabalenguas y las jitenjáforas que se adjuntan, han sido trasmitidas a la autora por tradición oral. Volumen 6, Número 3, Año 2006, ISSN 1409-4703 15 
- El docente canta la primera frase y los estudiantes la repiten en eco (escuchan la frase musical y después la repiten de igual manera).

- Se procede de la misma forma con el resto de frases hasta terminar el canto.

- Se invierte el procedimiento, ya que los estudiantes inician el eco.

- Posterior al eco se trabaja el canto mediante el uso de preguntas y respuestas

- La población estudiantil inicia las preguntas y respuestas

- Se procede a cantar completa la canción sin ayuda del docente.

- Se pueden utilizar todas las estrategias metodológicas antes descritas

\subsection{Lecto-escritura}

Esta área comprende la lectura y la escritura de la música. Existen varios códigos rítmicos que son útiles para la aplicación con la población estudiantil; entre ellos se puede citar "corro y voy" del método Martenot, el "dale y dan" de Ward y el "titi, ta" de Kodaly. En estos casos, las sílabas mencionadas se aplican a las negras y a las corcheas, sin embargo, existen otros términos para otras células rítmicas, que se utilizan para facilitar la percusión, la lectura y la interiorización de los diferentes valores musicales.

No obstante, es apropiado también incursionar en la lectura y la escritura no convencionales, cuyas partituras en realidad están formadas por símbolos, dibujos, gráficos o indicaciones específicas sobre los instrumentos que se van a utilizar en la obra, los ritmos, cambios de velocidad o intensidad, efectos vocales o sonidos corporales, u otras indicaciones sonoras interesantes.

En el apartado siguiente, se muestran ejemplos de partituras no convencionales, que facilitan los procesos de enseñanza y aprendizaje de la lectura y escritura de la música.

\subsection{Ejecución instrumental}

Posterior al trabajo corporal, los instrumentos de percusión (se golpean, se frotan, se sacuden para producir sonido) permiten vivenciar estos componentes rítmicos de manera independiente, o al realizar una banda rítmica. En este caso se manipulan maracas, claves, panderetas, panderos, tambores, chin chin $u$ otros, que se utilizan para percutir los componentes del ritmo, dependiendo de sus características específicas; es decir, la sonoridad, la vibración, la duración del sonido que emiten y la precisión rítmica entre otros. 
En esta área es interesante realizar ejercicios de "móviles sonoros", que consisten en improvisaciones musicales cuya partitura está representada por gráficos. Esta práctica estimula la creatividad entre los integrantes de la banda rítmica, ya que cualquiera puede convertirse en el director del grupo y dar su toque personal, a la vez que pueden surgir muchas posibilidades sonoras, dependiendo de las combinaciones tímbricas que cada director deseé dar. En este caso el trabajo es muy libre y variado, y no se requiere de una lectura musical rigurosa y técnica, por el contrario facilita la espontaneidad entre los integrantes. Un ejemplo de una "partitura de un móvil sonoro" puede ser la siguiente:

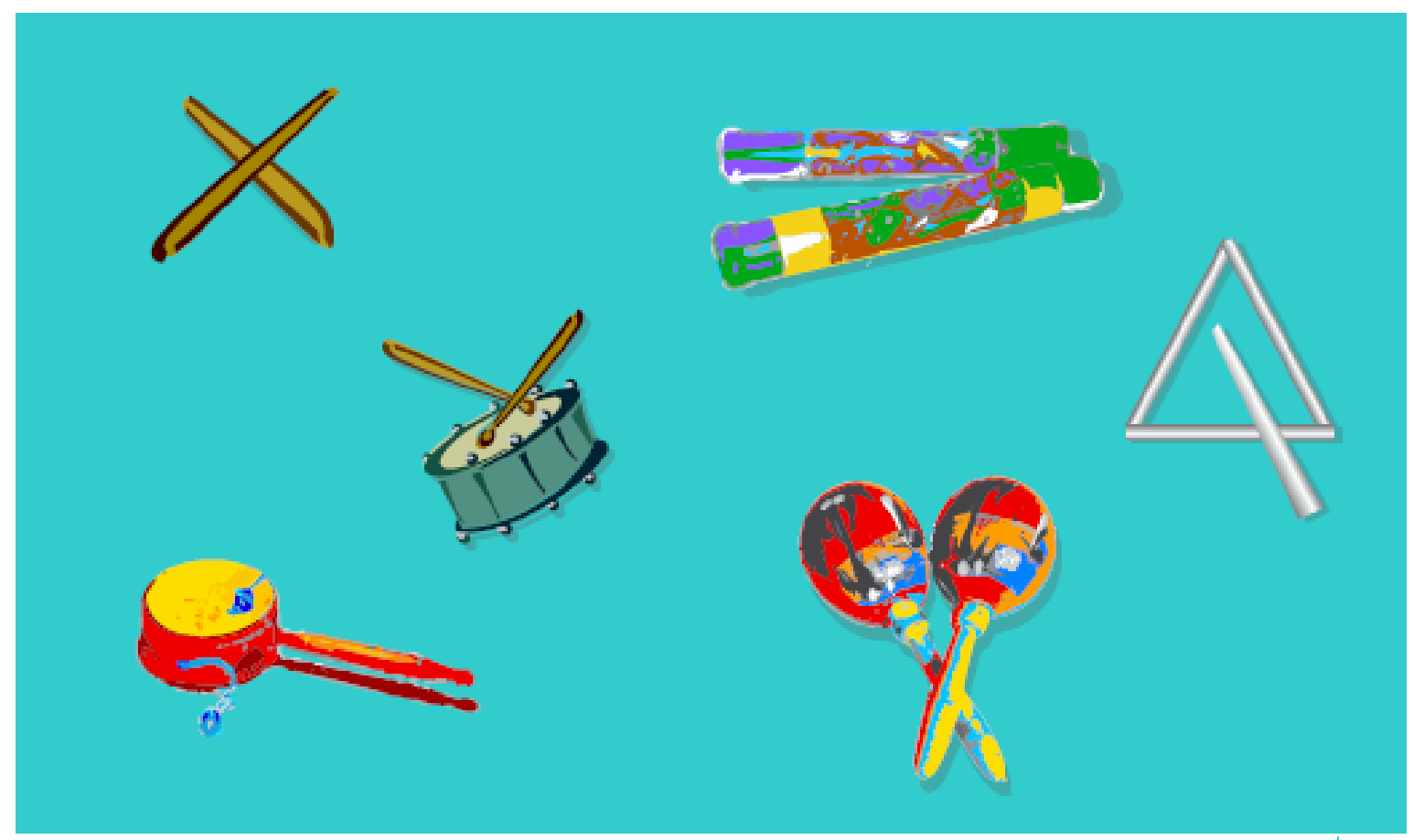

Además de los "móviles", la ejecución instrumental se puede trabajar con partituras no tradicionales, es decir, no se necesita de pentagramas para interpretar una creación musical. Por ejemplo 

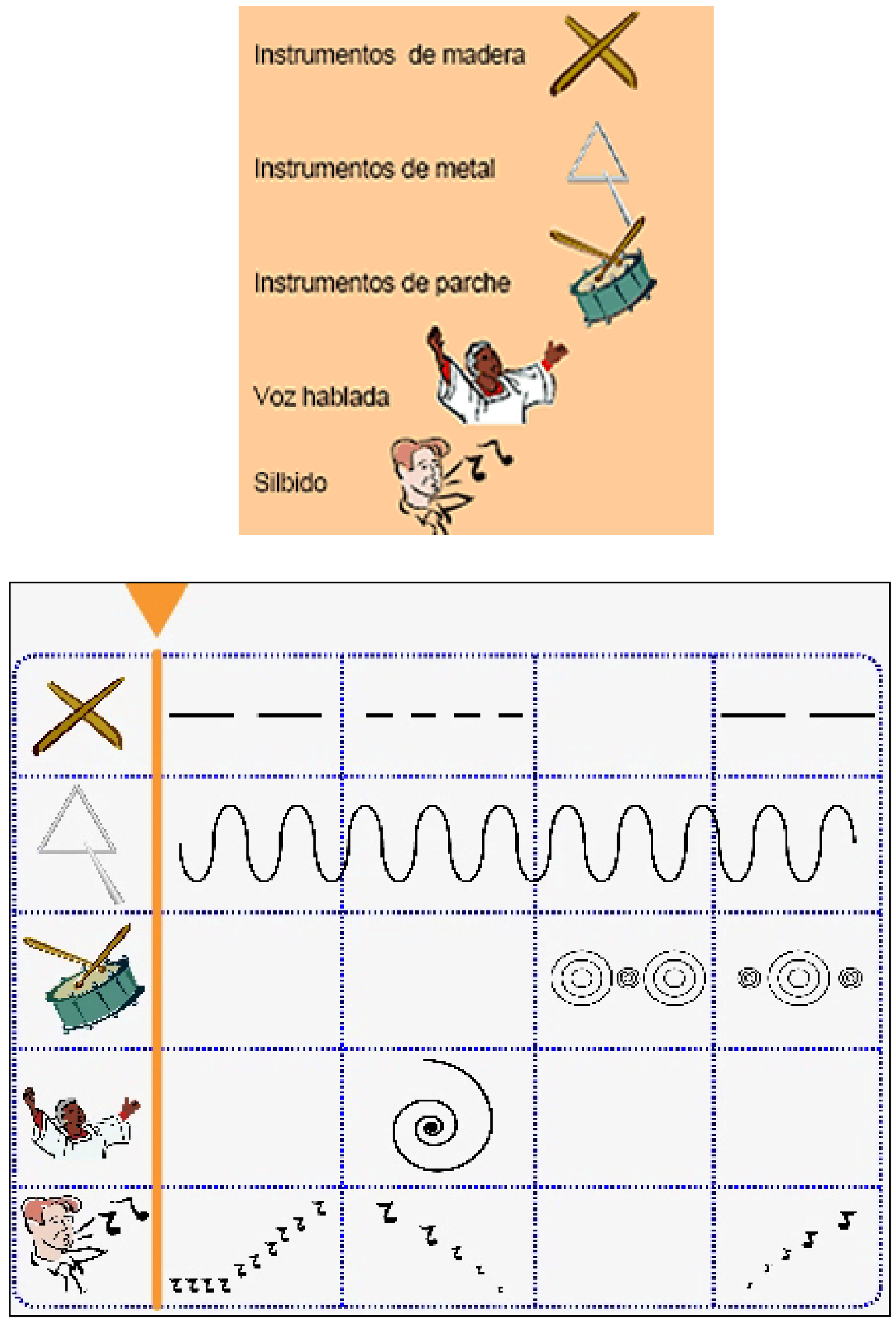
Posterior o paralelo a la experiencia de los "móviles sonoros", la ejecución instrumental ofrece también la posibilidad de realizar banda rítmica con partituras tradicionales. En ambas posibilidades, se pueden incluir instrumentos de percusión, de viento (se necesita una columna de aire para que se produzca el sonido), o de cuerda (es necesaria la vibración de cuerdas con diferentes grados de tensión).

La ejecución instrumental favorece el respeto, la cooperación, el "esperar turno", (aspecto importante de resaltar en las niñas y niños pequeños, que quieren ser atendidos siempre de primeros), estimula las relaciones interpersonales, ayuda a descargar tensiones, incrementa la responsabilidad y el orden, a la vez que favorece la recreación.

\subsubsection{Elaboración de instrumentos musicales con materiales de desecho}

Si bien es cierto el comercio ofrece una gran variedad de instrumentos musicales, también es conveniente conocer algunas ideas para confeccionarlos utilizando materiales reciclables, ya que abarata el costo de los mismos, se estimula la creatividad y el deseo por la ejecución de los mismos, asimismo, los estudiantes cuidan más los materiales que ellos y ellas han realizado.

Para la elaboración de los instrumentos con materiales de desecho, es conveniente tener en cuenta el peso, el tamaño y la confección de los mismos, ya que ninguno de ellos puede convertirse en un objeto peligroso para quienes lo utilicen. Por lo tanto se sugiere tomar las medidas necesarias para su confección.

Se adjuntan algunas ideas para la construcción de instrumentos y materiales musicales con elementos reciclables:

\section{- Claves Reproducir sonido}

Se necesitan dos pedazos de palo de escoba del mismo grosor y tamaño $(15 \mathrm{~cm}$.), lijados y sin pintar o barnizar (excepto que ya estuvieran pintados).

Este instrumento ofrece la posibilidad de percutir el pulso, el acento, el ritmo y el ostinato, ya que su ejecución es muy precisa. 


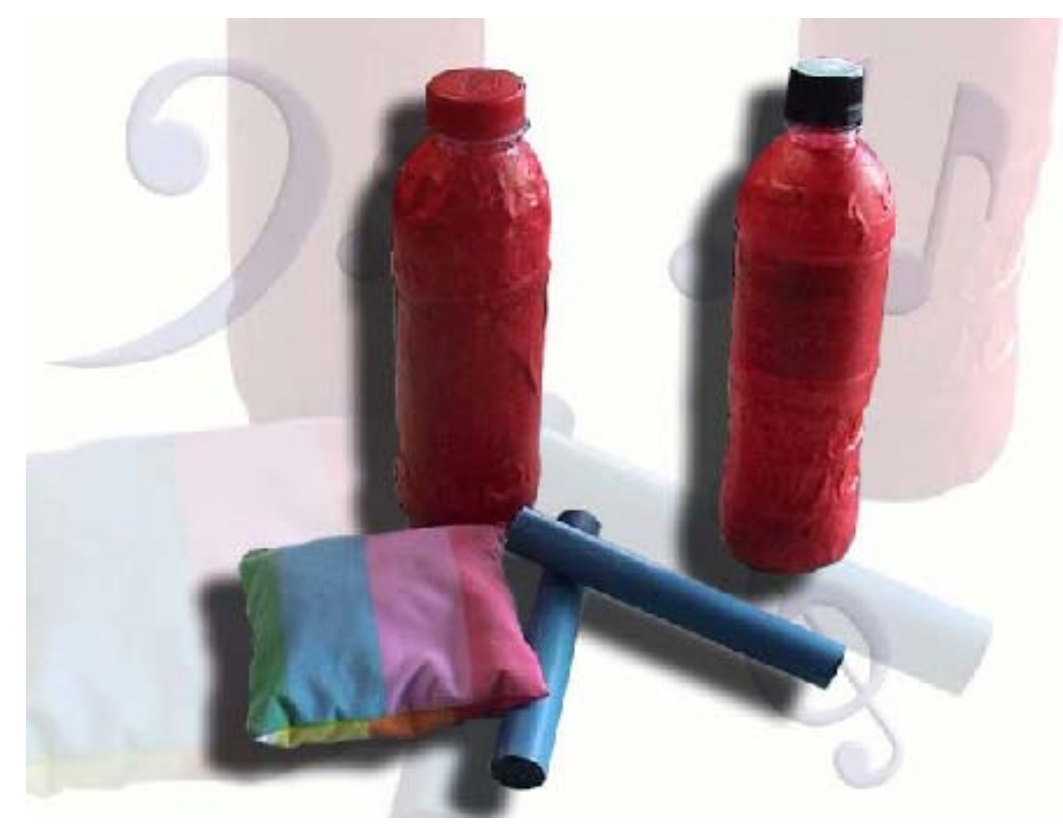

- Maracas Reproducir sonido

Se confeccionan con envases plásticos que se rellenan aproximadamente por la mitad con semillas o granos. Es conveniente elaborar dos de ellas de igual tamaño pero con granos diferentes que permitan emitir sonidos distintos (uno grave y otro agudo), al mismo tiempo, se necesita que no se vea el contenido de cada una de ellas, ni que tengan olores penetrantes o propaganda. En este último caso, es conveniente una decoración agradable pero no excesiva; no debe convertirse en un juguete.

Se aconseja utilizarla para marcar acento y ostinato.

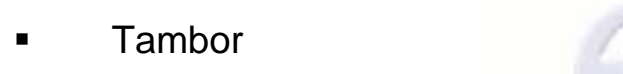


Se confecciona con envases plásticos de tamaño mediano o grande, al que se le pega una cinta para colgarlo; conviene que no contenga ningún olor fuerte ni propaganda comercial, por lo que es necesario decorarlo de manera discreta.

Los bolillos se hacen con dos lápices o bolígrafos sin tinta, a los que se les adhieren bolas pequeñas de hule, cinta adhesiva resistente, lana o estereofón.

Con él se ejecuta el pulso, el acento, el ritmo y el ostinato. Sin embargo, como su sonoridad es fuerte, se recomienda tener en cuenta el número de ellos que se utilizarán en el aula.

- Instrumento creado Reproducir sonido

Confeccionar algún instrumento de percusión, de viento o de cuerda con materiales de desecho.

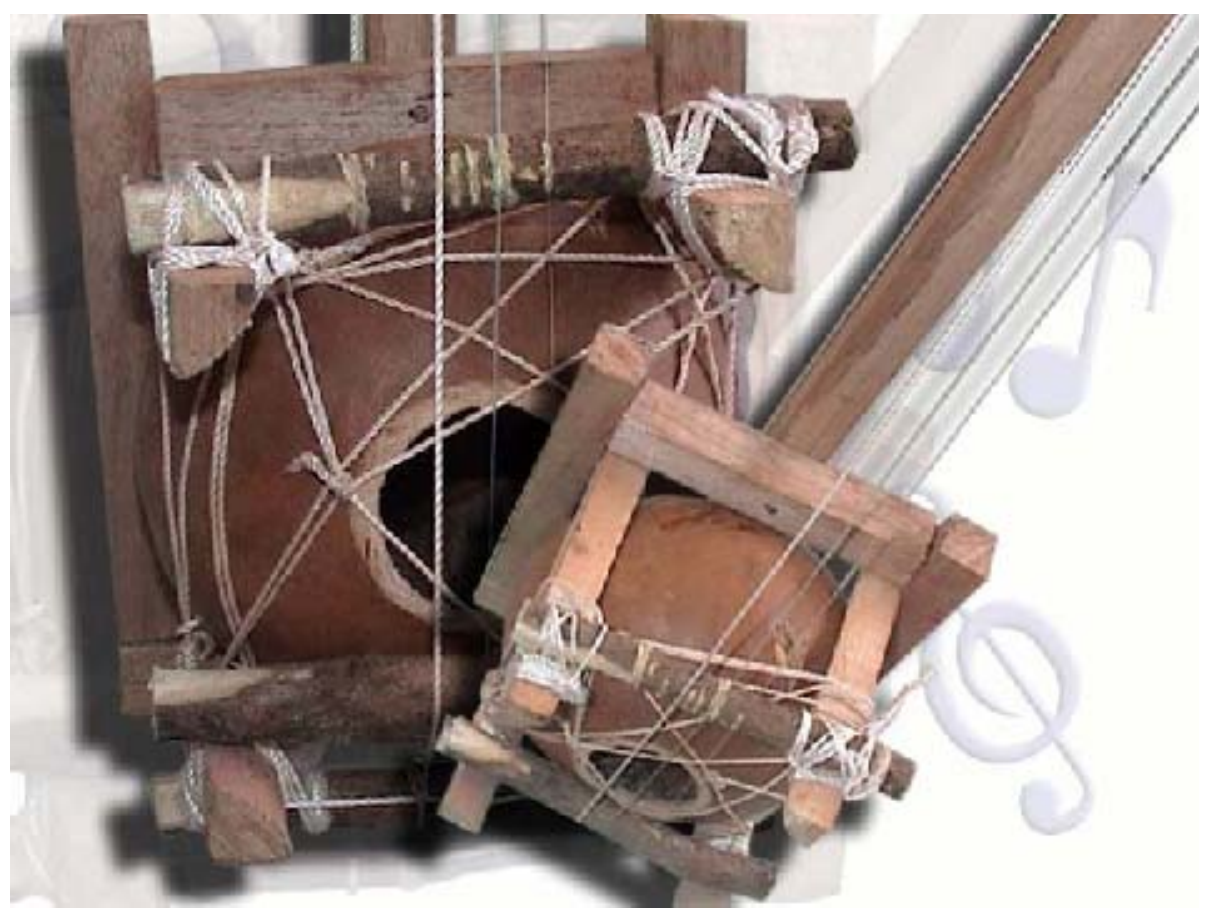




\section{- Triángulo Reproducir sonido}

Se elabora con varilla de construcción doblada, también puede utilizarse una herradura o una rejilla de baño. Cualquiera de las opciones anteriores se cuelga de un hilo de nylon y se golpea con una varillita de metal.

- Chin-chin Reproducir sonido

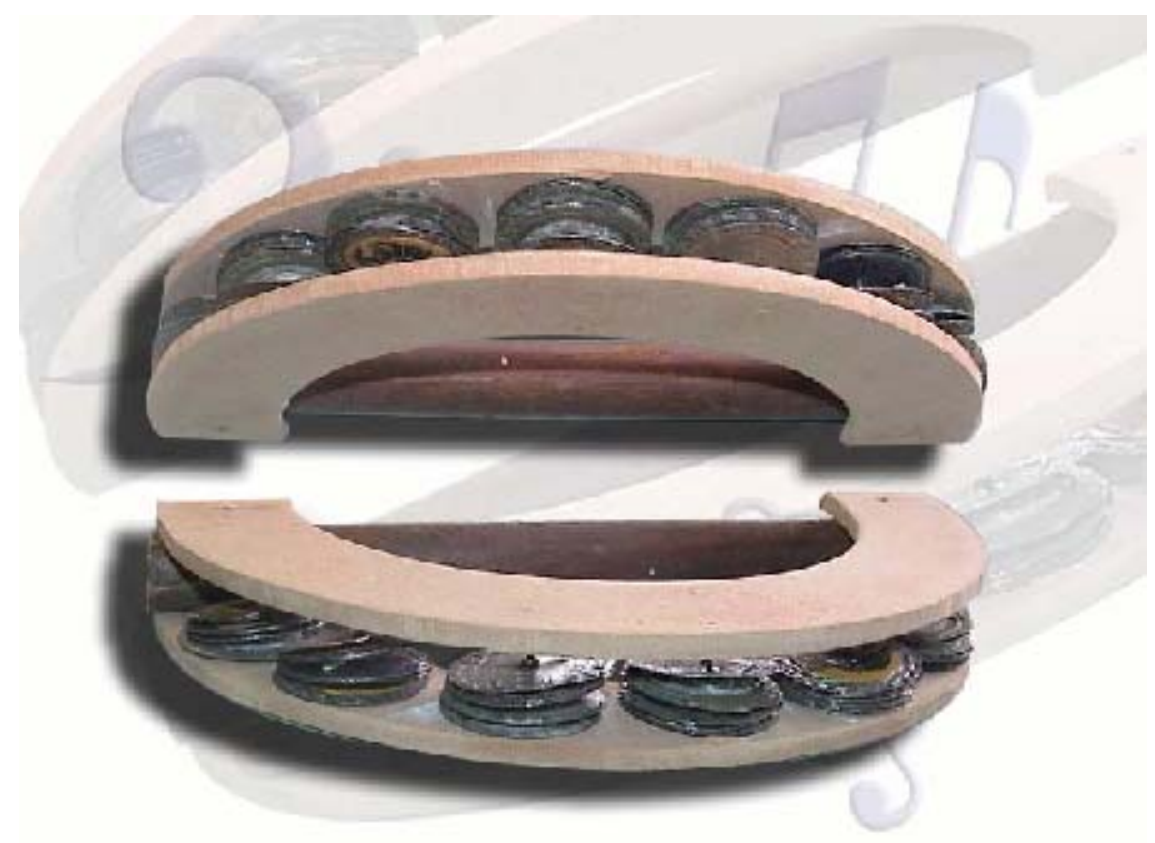

Se construye con una cuchara de cocina de madera, a la que se le adhieren clavos con chapas previamente aplastadas. Es importante la distancia entre cada uno de ellos y la cantidad de chapas que se coloquen, ya que si son demasiadas o están muy juntas, no permite que se muevan con facilidad y por lo tanto que vibren bien.

No conviene que el clavo salga por detrás (si es así se sugiere cortar o lijar), así como tampoco, que quede mucho espacio sin chapas; es decir el clavo que se utilice no puede ser demasiado grande. 


\subsubsection{Otras posibilidades de materiales sonoros}
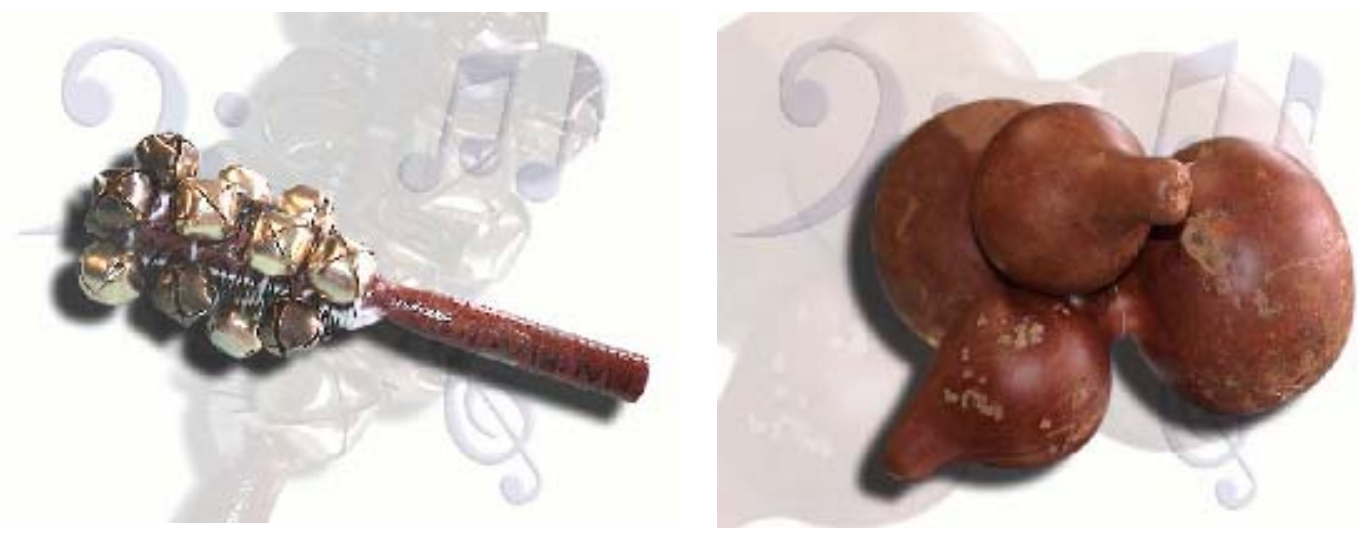

Mediante el reciclaje de materiales y la estimulación de la creatividad, se obtienen materiales sonoros diferentes, de fácil manipulación para la población estudiantil y de bajo costo. A continuación se mencionan algunos de ellos:

- Camino sonoro

Este material se elabora con dos vasos plásticos, a los cuales se les hace un agujero en sus bases. Por los orificios se les introduce una cuerda y en el otro extremo de la misma, se les ata un trozo de palillo de dientes. Los vasos se decoran con papeles de colores o se pintan.

La manera de utilizar el camino sonoro, es colocando el vaso en la boca para conversar, mientras la otra persona lo sitúa en la oreja para escuchar el mensaje.

\section{- Móvil silencioso}

Para construirlo se le hacen agujeros a las puntas del cañón de varias plumas y se les introduce un trozo de hilo de nylon (entre 25 y 20 centímetros de longitud,) a cada una, que es anudado para que no se suelten. El otro extremo de las cuerdas se sujeta a un pedazo de madera, dejando un trozo para que cuelgue. Conviene que cada cuerda quede separada una de la otra, para que no se enreden entre sí al moverse. De esta forma se convierte en un móvil sin sonido y se vivencia el silencio musical. 
- Móvil sonoro

Para confeccionar este material se necesitan fideos de cubitos que se les introduce un trozo de hilo de nylon (de unos 30 centímetros de longitud); debe quedar un espacio aproximado de 6 centímetros sin fideos, para introducirlo a un agujero que se le ha hecho a una tapa de cartón (se anuda para que no se suelte).

Se requieren varias tiras de fideos, colocadas cerca una de la otra, para que al moverse choquen entre sí y se produzca el sonido.

- Paisaje sonoro de una tormenta

Para los efectos sonoros de este paisaje, resultan útiles las bolsas de basura; depende de cómo se manipulen y del estado en que se encuentren.

Por ejemplo: si se sacuden con la abertura hacia arriba simulan los truenos, si se frotan imitan el sonido de la lluvia, si se mueven con suavidad será el sonido del viento y si se sujeta la bolsa desinflada entre las manos dándole golpecitos, produce un sonido similar al saltar sobre charcos.

- Paisaje sonoro de la playa

Para recrear el sonido del agua se remueve ésta con los dedos.

Para imitar el graznido de las aves, se le hace una abertura a lo largo de un envase plástico para guardar rollos de fotografía, y se perfora la base del mismo. Por este agujero se introduce una cuerda resistente; al final de la cuerda se le ata un pedacito de palillo de dientes y se le pega a la tapa del recipiente. Puede decorarse con papeles de colores

Para producir el sonido se sostiene el extremo del hilo que queda fuera del envase y se le da vueltas a diferentes velocidades para obtener sonidos distintos.

Si se desea simular el sonido de la sirena de un barco, se colocan los labios sobre el borde de una botella vacía y se sopla.

Otros paisajes sonoros pueden ser el de la escuela, el mercado, el bosque, un estadio y muchos más; para realizarlos se necesita descubrir los materiales adecuados para producir cada efecto sonoro, ya sea con sonidos corporales, mediante objetos, elementos de la naturaleza o acciones; todo depende de la curiosidad y del ingenio de los participantes.

Volumen 6, Número 3, Año 2006, ISSN 1409-4703 


\section{- Kelontong o monkey drum Reproducir sonido}

"Es un instrumento tradicional de la India, el Tibet y Vietnam. Su caja de resonancia es pequeña y estrecha, con una membrana a cada lado y dos bolitas unidas por un hilo cada una". (Vivamos la música, 2002, p. 47).

La manera de construirlo es haciéndole un pequeño corte en el borde de las dos caras de una caja. A una de ellas se le introduce un palito de madera que se le fija con cinta adhesiva (ambas se pueden decorar con pinturas, lápices de color, crayolas o marcadores). Luego se ata un botón a cada extremo de un trozo de hilo grueso. Éste se pega en el interior de una de las tapas; después se unen ambas tapas y se pegan también con la cinta adhesiva.

Para hacerlo sonar se sujeta con una mano y se le da vueltas, de forma que los botones percutan la superficie de las dos caras de la cajita. También se puede hacer rodar el mango entre las dos manos.

- Mirlitón

El efecto que produce este material consiste en modificar el timbre de la voz.

La manera de confeccionarlo es con un trozo de manguera de unos 15 o 20 centímetros de largo, al que se le hace un agujero de forma rectangular con un cutter o una cuchilla. Luego, se coloca un pedazo de papel de seda en el extremo más cercano al agujero y se sujeta con una liga.

Para hacerlo sonar se habla o se canta de manera normal, colocando el instrumento delante de la boca.

\section{- Agitófono Reproducir sonido}

Con un cutter o cuchilla se corta la base de tres botellas. Luego, se recorta uno de los envases en tiras verticales de 1.5 o 2 centímetros. Las otras botellas se recortan en tiras a diferentes alturas, para obtener sonidos distintos.

Para manipularas se sugiere sostener dos o tres botellas en cada mano y agitarlas para producir el sonido. 
- Lira

A una caja de cartón duro y de forma rectangular, se le recorta un círculo de 10 o 12 centímetros de diámetro, en el centro de uno de los lados. De preferencia el lado que sea escoja, conviene que sea alguno de los grandes, ya que esto ayudará en la calidad del sonido y también para manipular la lira.

Se procede a pegar un trozo de madera con cola de zapatero de 0.5 centímetros de grosor, 10 de longitud y 2 de altura aproximadamente, debajo del orificio que se le ha hecho a la caja. Posteriormente se colocan unas tres o cuatro ligas alrededor de la caja, de modo que queden situadas encima del trozo de madera y de forma equidistante. Para hacerla sonar sólo hay que rasgar las ligas.

\section{- Botella sonora}

Este instrumento se elabora con una botella plástica de gaseosa y un trozo de manguera de mayor tamaño que el del envase. El recipiente se llena de agua unas dos terceras partes y se le introduce la manguera al mismo tiempo que se sopla; dependiendo de lo que se meta o saque ésta de la botella, se obtienen diversos sonidos.

\subsection{Apreciación musical}

Conviene estimular a la población estudiantil para que tengan un abanico amplio de audiciones, donde se incluyan obras de distintos compositores, géneros musicales y períodos. Si embargo, es importante recalcar en ellos y ellas, la diferencia que existe entre oír y escuchar, ya que el primero corresponde a un acto inconsciente e involuntario, donde no se analiza ni se siente la música; mientras que al escuchar media la concentración, que le permite a la persona expresarse de manera cognitiva, física y afectiva.

La audición específicamente musical es una respuesta más psicológica que fisiológica, que está condicionada por la inteligencia, la imaginación, las motivaciones, la afectividad y los sentimientos. Cada uno, en definitiva, escucha una música en función de sus coordenadas socio-culturales. (Pascual, 2002, p. 300).

El compositor Aaron Copland, citado por Pascual (2002, p. 3002), considera que al escuchar la música intervienen un plano sensual, el plano expresivo y el plano musical. 
El sensual implica disfrute por lo que se oye y se convierte en una especie de acompañamiento para otras tareas. En el expresivo, se manifiestan estados de ánimo, sentimientos y sensaciones diversas que con palabras son difíciles de enunciar. En este caso, las obras utilizadas son de carácter descriptivo, relacionadas con historias o para ilustrar escenas cinematográficas. En el plano musical además de incluir los anteriores, se analizan las cualidades y los elementos del sonido; es decir la melodía, el ritmo, el timbre, la textura, la forma y otros.

Al apreciar una obra musical se identifican elementos previamente conocidos, aunque sean elementales, es por esta razón que el repertorio debe programarse cuidadosamente.

Cualquiera plano que se estimule en la apreciación musical, conviene realizarla de manera activa con los y las estudiantes, teniendo en cuenta la duración de la obra, la selección del fragmento que se va a escuchar (en caso de que sea muy extensa), aplicar la motivación al inicio de la actividad, tener en cuenta el momento y el lugar en donde se lleva a cabo, así como el uso de una técnica apropiada para lograr mejores resultados.

Por esta razón, se puede recurrir a aspectos históricos del compositor, acontecimientos geográficos, culturales o anecdóticos. Otras posibilidades son la dramatización, el movimiento y la danza, así como también la expresión gráfica o musicograma que permite integrar la percepción visual de la audición, mediante figuras geométricas, líneas o colores. También es útil recurrir a la audición comparada de versiones modernas que se realizan a partir de obras clásicas, y las audiciones en donde se emplean medios audiovisuales.

\section{Conclusiones}

Es importante brindar a la población estudiantil estrategias metodológicas lúdicas que permitan vivenciar la expresión musical en las diferentes áreas de trabajo, mediante la creación de materiales auxiliares e instrumentos musicales con materiales reciclables.

Al experimentar con elementos sonoros surgen ideas musicales que se combinan y se ordenan bajo la percepción auditiva. De igual manera, las decisiones de qué hacer se toman ejecutándolas al instante y provocan placer al jugar e inventar con el sonido, en búsqueda de una interpretación cada vez más expresiva. (Arguedas, 2003, p.3) 
Pedagogos musicales como Orff, Dalcroze, Willems, Kodály, Martenot, Ward, Paynter, Suzuki, Schafer, Montessori, Violeta Hemsy y otros más, han enfatizado en aspectos específicos de la enseñanza musical. No obstante, consideran importante desarrollar cada uno de ellos, de manera progresiva mediante la vivencia y el sentido lúdico; su objetivo es estimular la sensibilidad, la creatividad, el canto, el ritmo, la audición la ejecución y el movimiento. Proponen que la educación musical sea un elemento formativo, más que una asignatura especializada, y se preocupan porque trascienda el entorno escolar, para musicalizar a la sociedad.

La experimentación de los elementos de la música mediante la manipulación de instrumentos y objetos sonoros y la vivencia corporal, son indispensables para realizar un aprendizaje por descubrimiento. Por este motivo es indispensable ofrecer la mayor cantidad de oportunidades, para que la población estudiantil esté en contacto con la expresión musical, ya sea con cantos, bandas rítmicas, juegos auditivos, movimientos y percusiones corporales o improvisaciones, ya que de esta manera se pretende despertarles sus facultades musicales.

Martín (1999, p. 25), considera que la actividad musical no requiere de aptitudes especiales y no es privilegio de unos pocos, por el contrario, la musicalidad siempre existe en las personas en diferentes grados, por lo tanto es indispensable potenciarla y desarrollarla.

Para poder realizar esta motivación entre las y los estudiantes, se necesita una actitud diferente de los docentes ya sean especialistas en educación musical, docentes de las materias básicas o de otra área del currículo escolar. En el profesorado debe prevalecer, el deseo de brindar estrategias que inviten a "hacer música" en todo momento del período lectivo, pues ésta siempre será una estrategia integradora y lúdica para cualquier contenido escolar.

Las propuestas de trabajo deben integrar aspectos cognitivos, psicomotores y afectivos, de una manera lúdica y comunicativa. Es importante fomentar la sensibilidad, el deseo del descubrimiento y la toma de conciencia hacia el entorno sonoro, así como también, hacer valorar a la población estudiantil sus creaciones, para estimular el gusto, el respeto hacia los demás y el sentido crítico. 
Conviene reflexionar sobre el hecho de que los seres vivos estamos inmersos en un mundo sonoro. En él, las personas tenemos la misión de cuidarlo, evitando la contaminación sónica y dando prioridad a la musicalidad que nos ofrece la naturaleza.

De igual manera, al encontrarnos rodeados de estímulos sonoros, vale la pena experimentar con ellos, pues todo ser humano en mayor o menor grado, es músico; en algunos casos sólo hace falta el estímulo adecuado.

La música se aprende haciendo música, la reflexión conceptual y teórica viene después de la vivencia. Las niñas y los niños aman y disfrutan la música, porque el mundo sonoro los ha acompañado desde el vientre materno, no es ajeno a su realidad; necesitan "hacer música" constantemente.

¡Docentes, los invito a vivenciarla en su trabajo de aula, ya que es una herramienta imprescindible!

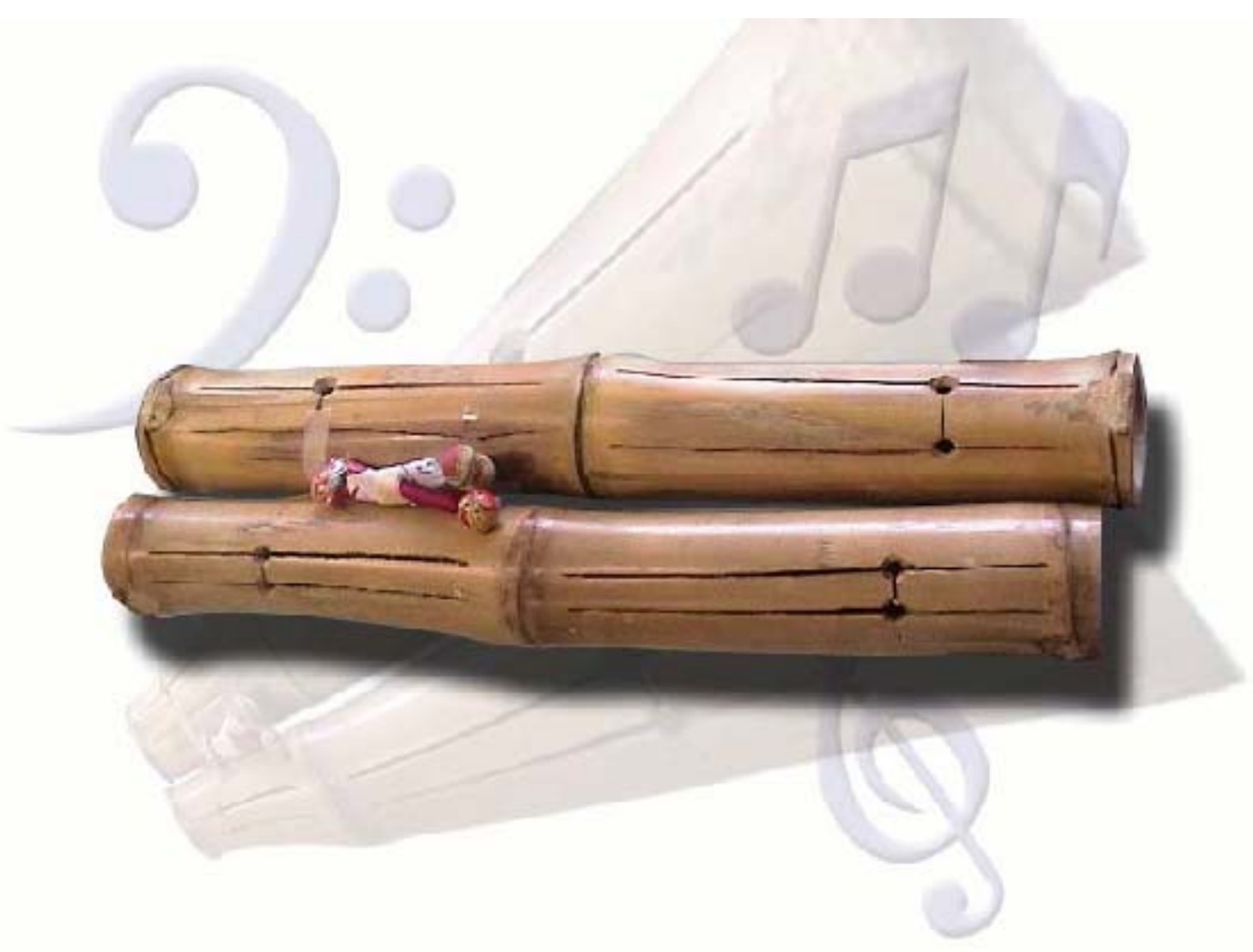




\section{Referencias}

Alsina, Pep, et al. (1999). La música en la escuela infantil. Didáctica de la música. La música en la educación infantil. Eufonía (14), 5-8.

Arguedas, Consuelo. (2003). La improvisación musical y el currículo escolar. Revista Electrónica Actualidades Investigativas en Educación, 3 (2). Disponible en http://revista.inie.ucr.ac.cr

Betés de Toro, Mariano. (2002). Fundamentos de musicoterapia. Madrid: Ediciones Morata S.L

Real Academia Española. (1984). Diccionario de la lengua española (20a. ed., Tomo 1). Madrid, España: Editorial Espasa-Calpe, S.A.

Glover, Joanna. (2004). Niños compositores (4 a 14 años). España: Editorial GRAÓ.

Martín, Conchita. (1999). La música en la escuela infantil. Didáctica de la música. La música en la educación infantil. Eufonía (14), 24-33.

Mejía, Pascual. (2002). Didáctica de la música. España: Prentice May.

Muñoz, Juan Rafael. (2001). La voz y el canto en la educación infantil. Didáctica de la música. En torno a la voz humana. Eufonía (23), 43-54.

Vivamos la música. (2002). La vida tiene música (Vol. 1, 2, 3, 4). Barcelona, España: Parramón Ediciones S.A. 